\title{
Reference frames and haptic perception of orientation: Body and head tilt effects on the oblique effect
}

\author{
MARION LUYAT \\ University Charles de Gaulle, Villeneuve d'Ascq, France \\ EDOUARD GENTAZ \\ University René-Descartes, Boulogne-Billancourt Cedex, France \\ TONY REGIA CORTE \\ University of Lille, Villeneuve d'Ascq, France \\ and \\ MICHEL GUERRAZ \\ Institute of Neurology, National Hospitalfor Neurology and Neurosurgery, London, England
}

\begin{abstract}
The aim of this study was to examine the effect of body and head tilts on the haptic oblique effect. This effect reflects the more accurate processing of vertical and horizontal orientations, relative to oblique orientations. Body or head tilts lead to a mismatch between egocentric and gravitational axes and indicate whether the haptic oblique effect is defined in an egocentric or a gravitational reference frame. The ability to reproduce principal (vertical and horizontal) and oblique orientations was studied in upright and tilted postures. Moreover, by controlling the deviation of the haptic subjective vertical provoked by postural tilt, the possible role of a subjective gravitational reference frame was tested. Results showed that the haptic reproduction of orientations was strongly affected by both the position of the body (Experiment 1) and the position of the head (Experiment 2). In particular, the classical haptic oblique effect observed in the upright posture disappeared in tilted conditions, mainly because of a decrease in the accuracy of the verticaland horizontal settings. The subjective vertical appeared to be the orientation reproduced the most accurately. These results suggest that the haptic oblique effect is not purely gravitationally or egocentrically defined but, rather, depends on a subjective gravitational reference frame that is tilted in a direction opposite to that of the head in tilted postures (Experiment 3).
\end{abstract}

This work addresses the question of the reference frame in which spatial orientations are defined in the haptic (tactual-kinesthetic) system of humans. Previous experiments have shown that orientation processing differs according to the value of the orientation. Indeed, the vertical and horizontal orientations are perceived more accurately than the oblique orientations. This anisotropy, called the oblique effect by Appelle (1972), is present in both the visual and the haptic systems. In the visual system, this effect has been demonstrated in a wide variety of tasks (detection, discrimination, recognition, and memorization; for reviews, see Appelle, 1972; Essock, 1980; Gentaz \& Ballaz, in press; Howard, 1982). In the haptic system, the

This work was supported by grants from the University Pierre Mendès France of Grenoble (CNRS EP 617) and the "Cognitique" Program(University René-Descartes, Paris V, and University Charles de Gaulle, Lille $3)$. We thank Yvette Hatwell for her helpful comments on an earlier version of this manuscript. We thank Daniel Gola for his technical support. Correspondence and requests for reprints should be sent to M. Luyat, Laboratoire URECA, UFR de Psychologie, Université Lille 3, Pont de Bois, BP 149, 59653 Villeneuve d'Ascq, France (e-mail: luyat@univ-lille3.fr). oblique effect has been studied in only one type of task: an exploration-reproduction task in which the blindfolded subjects were asked to explore a rod with one hand and to reproduce its orientation with the same or the contralateral hand (Appelle \& Countryman, 1986; Gentaz \& Hatwell, 1995, 1996, 1998, 1999; Lechelt, Eliuk, \& Tanne, 1976; Lechelt \& Verenka, 1980). Although the explanations of these visual and haptic oblique effects are still debated (Gentaz, 2000; Gentaz \& Ballaz, in press; Heeley \& Buchanan-Smith, 1990; Heeley, Buchanan-Smith, Cromwell, \& Wright, 1997; Saarinen \& Levi, 1995), this anisotropy suggests that orientations could be coded relative to a reference frame in which the vertical and horizontal orientations would be the reference norms (Cecala \& Garner, 1986; Gentaz, 2000; Gentaz \& Hatwell, 1996; Gentaz et al., in press). In this perspective, identifying the nature of the spatial reference frame in which orientations are mapped would allow a better understanding of these oblique effects.

Different reference frames can be selected to define an orientation in space. A classical broad distinction is made 
between egocentric (referred to the subject's body) and allocentric (referred to environmental cues) spatial frames (for reviews, see Howard, 1982; Rock, 1990; N. J. Wade, 1992). Several egocentric reference frames can be defined: (1) a retinocentric reference frame specifically relevant for visual tasks, (2) head- or trunk-centered reference frames implied in both visual and haptic tasks, and (3) a hand- and shoulder-centered reference frame that can be relevant for haptic tasks. The allocentric reference frame can be divided into a gravitational frame defined by the direction of the pull of gravity (geocentric frame) and pattern-centric reference frames defined by visual or haptic contextual cues. In natural conditions, the gravitational, body-centered, and visual reference frames are most often aligned. Consequently, the visual and haptic oblique effects might result from either egocentric or allocentric mapping of orientations. However, in the absence of visual or tactual contextual cues, tilting the head or the body uncouples the gravitational and the egocentric reference frames, allowing us to specify in which reference frame orientations are defined.

In vision, the effect of head or body tilt on the visual oblique effect has been investigated with different tasks, such as visual search (DeFord, Prather, \& Essock, 1998, 1999), orientation-discrimination (Attneave \& Olson, 1967; Buchanan-Smith \& Heeley, 1993; Chen \& Levi, 1996; Ferrante, Gerbino, \& Rock, 1995; Orban, Vandenbussche, \& Vogels, 1984), contrast sensitivity (Banks \& Stolarz, 1975; Lennie, 1974), and vernier acuity (Corwin, Moskowitz-Cook, \& Green, 1977; Saarinen \& Levi, 1995). Some experiments using orientation-discrimination tasks showed results in favor of a retinal reference frame (Chen \& Levi, 1996; Orban et al., 1984). In these cases, the most accurately processed orientations were those aligned with the vertical and horizontal retinal meridians, whatever the position of the head. Thus, the visual oblique effect could be located at a low level of visual processing (Furmanski \& Engel, 2000), where the retinotopical mapping of most orientation-selective neurons is preserved. By contrast, other studies showed results in favor of a gravitational reference frame (Attneave \& Olson, 1967; Buchanan-Smith $\&$ Heeley, 1993; Ferrante et al., 1995), suggesting the involvement of nonvisual cues, such as vestibular and somesthetic ones. These latter results were consistent with a high level of visual processing of orientation-in particular, a level at which orientation constancy could be achieved.

Most of these contradictory results could be explained by the experimental paradigm used as suggested by Essock (1980). Indeed, Essock has proposed a distinction between two classes of oblique effects, depending on the nature of the paradigm. The Class 1 oblique effects, obtained in paradigms measuring the basic functioning of the visual system (e.g., acuity, contrast threshold), would be tied to retinal coordinates. The Class 2 oblique effects, observed in paradigms reflecting cognitive processes (such as identifying, remembering, or categorizing the orientations of stimuli), could be tied to another reference frame, such as the gravitational reference frame.
Chen and Levi (1996) have pointed out other factors that could explain the apparent inconsistency in the studies using orientation-discrimination tasks: (1) The countertorsion of the eyes (see Bles \& de Graaf, 1991; de Graaf, 1990) provoked by body or head tilt, which could lead to an apparent gravitational mapping if it is not taken into account, (2) the length of the stimuli (long stimuli could enhance a retinal reference frame), (3) the tilted segment (tilting the head alone while keeping the trunk aligned with gravity could favor a gravitational mapping), and (4) the presence of an explicit spatial reference (parallel or perpendicular line) during the task, which could enhance a retinal reference frame.

In haptics, as was noted by Essock, Krebs, and Prather (1997), the effect of body tilt on the oblique effect has not yet been investigated. However, in a connected field, Van Hof and Lagers-van Haselen (1994) have studied the effect of body tilt on orientation-discrimination by the proprioceptive system. In this experiment, the authors asked sitting blindfolded subjects to grasp two rods simultaneously, one with each hand, and to judge whether these rods were parallel or not. Four orientations were studied (vertical, horizontal, $45^{\circ}$ and $135^{\circ}$ egocentrically defined orientations) in two postural conditions (body erect and body tilted $45^{\circ} \mathrm{left}$ ). The results favored a gravitational reference frame, since more accurate performances were observed in the gravitationally defined vertical and horizontal orientations than in the gravitationally defined oblique orientations, whatever the body position. However, one result found of their experiment, which they did not discuss, was that tilting the body increased the threshold of discrimination, especially for the gravitationally defined vertical and horizontal orientations. This result would be difficult to explain if orientations had been encoded in a purely gravitational frame. Actually, the deviation of the apparent vertical provoked by head or body tilts could explain this result.

In darkness, head or body tilts are known to induce constant deviations and to decrease the precision of the perception of the physical vertical (for a review, see Howard, 1982). Adjustments are displaced either in the direction of the postural inclination (the Aubert or A-effect) or in the opposite direction (the Müller or E-effect). The direction of the subjective vertical (SV; A- or E-effects) depends on the magnitude of tilt and also on the perceptual modality in which the rod adjustments are made (Guerraz, Luyat, Poquin, \& Ohlmann, in press; Luyat, 1997). Although very few experiments have been carried out in the haptic modality, body or head tilts seem to give rise to systematic E-effects in this modality (Balliet \& Nakayama, 1978; Bauermeister, Werner, \& Wapner, 1964; Guerraz et al., in press). The origin of the A- and E-effects is unclear and is still debated (see S. Wade \& Curthoys, 1997). These effects have been related to a misperception of the body, the A-effect resulting from an underestimation of body tilt and the E-effect from an overestimation of body tilt. Four main explanatory factors have been proposed by Howard (1982): (1) the countertorsion of the eyes, which leads to a visual E-effect (overestimation of 
head tilt) if the system does not take into account the $10 \%$ countertorsion of the retinal meridians provoked by head tilt, (2) changes in vestibular inputs, particularly from the otoliths organs, (3) changes in somesthetic inputs arising from contact between the body and the surfaces that support it, and (4) changes in kinesthetic inputs from the joints and musculature of the legs, the back, and in the case of head tilt, the neck.

However, several authors have cast doubt on the assumption that A- and E-effects reflect a misperception of the body (Mittelstaedt, 1983; S. Wade \& Curthoys, 1997). According to Mittelstaedt's (1983) alternative model, the SV is calculated from a gravity vector transduced by vestibular and somesthetic receptors summed with vectors representing the orientation of the observer's own head and body axes (idiotropic vector). Thus, in the case of body tilt, the A-effect reflects the attraction of the SV by the idiotropic vector. In this model, the reverse deviation of the visual SV (the E-effect) has been related to a particular signal coming from the utricule and saccule -in particular, when the utricular signal is reduced relative to the saccular signal (see Mittelstaedt, 1995, and also Parker \& Poston, 1984). On the other hand, according to S. Wade and Curthoys, the deviation of the visual SV provoked by body tilt would be linked to the countercyclotorsion of the eyes. Whatever the factors involved in these tilt effects, A- and E-effects demonstrate that tilted subjects do not have access to a veridical gravitational reference frame but, rather, to a subjective gravitational reference frame that is no longer congruent with the physical one. In haptic modality, for example, a rod aligned with the physical vertical will be perceived as deviating in the direction of the head or body tilt, and as a consequence, the SV, in this case, will deviate in the direction opposite to the head or body. As a result, in a tilted posture, the gravitational vertical orientation could become an oblique orientation for the subject, and the SV could constitute the principal orientation.

The purpose of the present research was to study the nature of the reference frames in which orientations are mapped in the haptic perceptual system. Thus, we investigated the effect of whole-body tilt (Experiment 1) and head tilt alone (Experiment 2) on the haptic oblique effect by using the classical exploration-reproduction task. This haptic perception of orientations is different from the proprioceptive coding of orientations involved in Van Hof and Lagers-van Haselen's (1994) experiment. Indeed, the exploration-reproduction task requires scanning movements of the shoulder-hand system, whereas the proprioceptive task implied only a static coding of rod position. Gentaz and Hatwell (1996) have shown that the scanning shoulder-hand movements are involved in the haptic oblique effect because of the gravitational cues provided by the antigravitational forces developed during scanning. These cues could reinforce, in an upright posture, gravity direction as one axis of reference. Thus, we asked blindfolded subjects, either upright or tilted, to scan a rod with one hand and, after a short delay during which the initial position of the rod was modified, to reproduce the previous orientation with the same hand (the Class 2 oblique effect in Essock's 1980, terminology). The exploration and reproduction of the rod was carried out in the absence of tactual contextual cues.

\section{EXPERIMENT 1 Whole-Body Tilt Effects on the Haptic Oblique Effect}

The aim of this experiment was to study the effect of whole-body tilts on the haptic oblique effect. In the main exploration-reproduction task, blindfolded subjects were asked to explore a standard orientation in the frontoparallel plane and, after a 5-sec unfilled delay, to reproduce this orientation. Three postural conditions were investigated: standing upright body (UB), body tilted $45^{\circ}$ to the left (BL), and body tilted $45^{\circ}$ to the right (BR). Five different orientations, defined gravitationally, were proposed: vertical $\left(0^{\circ}\right)$, horizontal $\left( \pm 90^{\circ}\right), 45^{\circ}$ counterclockwise oblique $\left(-45^{\circ}\right), 45^{\circ}$ clockwise oblique $\left(+45^{\circ}\right)$, and the SV. A first phase estimated the SV of each subject in each postural condition (Phase 1), and this orientation was further tested in the exploration-reproduction task. Moreover, because S. Wade and Curthoys (1997) have suggested that the free scanning of the rod in the haptic modality was not a satisfactory method of assessing the perception of orientation, we measured the SV of each subject (Phase 2 ) again after the exploration-reproduction task. The procedures used in Phase 1 and Phase 2 were the same.

Three main hypotheses were formulated. If the oblique effect was defined in a gravitational reference frame, the reproduction of the vertical and horizontal orientations should be more precise than the reproduction of the oblique orientations $\left(+45^{\circ}\right.$ and $-45^{\circ}$ orientations), whatever the conditions of postural tilts. By contrast, if the haptic oblique effect was defined in an egocentric reference frame, the reproduction of the oblique orientations $\left(-45^{\circ}\right.$ and $\left.+45^{\circ}\right)$, which are parallel and perpendicular to the body in the tilted conditions, should be more precise than the reproduction of the gravitational vertical and horizontal orientations, now oblique relative to the body axis. However, given the strong body tilt effects on the perception of the vertical, the SV may constitute a reference axis used when the body is inclined. In this latter case, the reproduction of the SV should be more precise than the reproduction of all the other orientations in the tilted postural conditions.

\section{Method}

\section{Subjects}

The subjects were 8 right-handed students in psychology ( 4 females, 4 males; mean age, 26 years). Their handedness was assessed by Bryden's (1977) five-item hand preference questionnaire (writing, throwing, drawing, using scissors, and using a toothbrush). On the basis of self-report, the subjects had no hearing or vestibular disorders, including diseases with ocular manifestation or motion sickness. 


\section{Apparatus}

The haptic apparatus. The haptic apparatus was composed of a metal disk (diameter, $40 \mathrm{~cm})$ equipped with a $\operatorname{rod}(25 \times 1.8 \mathrm{~cm})$. This rod, mounted on the center of the disk, could be rotated $360^{\circ}$ around its central axis. Magnets were fixed inside the rod to maintain it in the desired orientation and to prevent involuntary deviation from its position during haptic scanning. A small amount of force was required for its position to be changed intentionally. The rod was fixed directly in contact with the disk. The disk was graduated in degrees (the sensitivity threshold of this display was equal to $\left.0.25^{\circ}\right)$. The rod and the disk were positioned in the frontal plane and were centered on the midline of the subject's body in the upright condition. The height of the disk was adjusted with reference to the subject's height, so that the top of the disk was at the level of the subject's shoulders.

The tilted board. In the tilted positions, the subject lay on his or her side on a wooden board that was $192 \mathrm{~cm}$ long, $62 \mathrm{~cm}$ wide, and $2 \mathrm{~cm}$ thick. It included a footrest at the bottom. A solid framework supported it at a fixed position tilted $45^{\circ}$. In the $45^{\circ}$ left-tilted position, the subject lay on his or her left side, and in the $45^{\circ}$ right-tilted position, he or she lay on his or her right side on the board. A smooth carpet was fixed on the board, and a cushion was placed under the head of the subject in order to maintain it in line with the trunkthat is, at a $45^{\circ}$ tilt from the gravitational vertical.

\section{Experimental Conditions and Procedure}

The subject was taken individually into a quiet room, in which he or she was asked to wear a neckbrace in order to prevent head movements and an occluded dark mask on the eyes during the experiment. Each subject was examined in three sessions corresponding to the three postural conditions: $\mathrm{UB}, \mathrm{BL}$, and BR. There was about 1 week between the different sessions, and their order was randomized. In each session, each subject was tested in two kinds of tasks: (1) a production task (Phase 1 and Phase 2) and (2) an explorationreproduction task. In total, in one session, the procedure comprised three successive periods: (1) the production task (Phase 1), (2) the exploration-reproduction task, and (3) the production task (Phase 2). A delay of about 5 min was given between these periods.

Production task. The purpose of the production task was twofold. First (Phase 1 of the production task), this task estimated the value of the SV for each subject in each of the three postural conditions (UB, BL, and BR) in order to test further this subjective orientation in the exploration-reproduction task. Second (Phase 2), in order to test the stability and reliability of the subjective orientation, this production task was performed again by each subject, with exactly the same procedure, after the main exploration-reproduction task had been completed.

In the production task, the subject was asked to adjust the rod to the physical vertical. A gravitational definition of the vertical was given: the direction of the rain without wind and the direction of a plumbline. During the adjustment, no time limit was imposed, but the subjects were told that hand movements should be restricted to the rod without touching the disk located below. Eight adjustments to the vertical were performed. The initial rod position was about $25^{\circ}$ away from the physical vertical, and the direction (counterclockwise and clockwise) was counterbalanced over trials.

Exploration-reproduction task. The aim of the explorationreproduction task was to investigate the classical haptic oblique effect and to test a body tilt effect on this phenomenon. In this task, a starting verbal signal of the experimenter asked the subject to scan the oriented standard rod (over its full length) haptically with one hand (i.e., to actively move the arm-hand-digit system), with no time constraint. The subject moved his or her hand off the rod when haptic exploration was completed. During the 5-sec unfilled delay, the subject was asked to maintain the hand in contact with his or her abdomen. During this delay, the experimenter modified the rod orientation by $\pm 25^{\circ}$ relative to the standard orientation used in the trial. The left/right direction from the standard orientation was counter- balanced between the eight trials performed for each of the five standard orientations tested. A verbal signal indicated the end of the delay and asked the subject to reproduce the orientation previously explored with the same hand (with no time constraint and without feedback).

Five standard orientations (defined gravitationally) were tested with eight trials each: the vertical $\left(0^{\circ}\right)$, the horizontal $\left( \pm 90^{\circ}\right)$, the $45^{\circ}$ left oblique $\left(-45^{\circ}\right)$, the $45^{\circ}$ right oblique $\left(+45^{\circ}\right)$, and the SV estimated previously in the production task and in the same postural condition. The order of presentation of these orientations was randomized for each subject. The rod was polarized; one of its extremities constituted the top, which pointed toward the standard value. For the $90^{\circ}$ horizontal standard orientation, it was in fact the $-90^{\circ}$ (top of the rod in the direction of the $-90^{\circ}$ value) that was tested and reproduced in the $\mathrm{UB}$ and the $\mathrm{BL}$ conditions. However, for the BR condition, since the left hand was used (see the Postural Conditions section), the $+90^{\circ}$ orientation was tested in order to keep the symmetry of exploratory movements between the BL and the BR conditions. The exploration-reproduction task consisted of 40 test trials ( 5 orientations $* 8$ trials). Prior to the experiment, a familiarization phase was proposed, during which the subject performed two trials randomly selected from the five standard orientations.

Postural conditions. As was mentioned before, each session corresponded to one of the three postural conditions: UB, BL, and BR. Each session consisted of 56 test trials: (1 orientation in the production task $* 2$ phases $* 8$ trials $)+(5$ standard orientations in the exploration-reproduction task $* 8$ trials). In total (in the three sessions, corresponding to the three postural conditions), there were 168 test trials per subject. The duration of one session was about $1 \mathrm{~h}$ (in total, $3 \mathrm{~h}$ per subject). During the UB session, the subject stood upright with his or her back against the wall and was told not to move his or her body and to hold it straight during adjustments (the right hand was used in this condition). A mark on the wall allowed us to control the eventual deviation of the head. During the bodytilted sessions, the subject lay on his or her side on the wooden board, which was tilted at $45^{\circ}$. The position of the head was exactly in the line of the body. The right and the left hands were used in the BL and BR postural conditions, respectively, so that similar (symmetrical) scanning hand movements were produced in the two bodytilted conditions.

\section{Results}

\section{Production Task: Stability and Reliability of the Measure of the Subjective Vertical}

For each trial, the algebraic deviation from the gravitational vertical was noted. According to convention, deviations to the left (rod turned counterclockwise) were counted as negative, and deviations to the right (clockwise) as positive. In order to determine the value of the haptic $\mathrm{SV}$ in each postural condition, the mean algebraic deviations (in degrees) over the eight trials in each postural condition were computed. Therefore, three SV estimations were obtained and used further in the explorationreproduction task as standard orientations in the corresponding postural condition.

In order to test the stability and reliability of the haptic $\mathrm{SV}$, we compared the performances obtained in Phase 1 of the production task (before the exploration-reproduction task) with those obtained in Phase 2 (after the explorationreproduction task) for the three postural conditions. Two main measures were computed: the mean signed errors (or constant errors; MSE) and the mean unsigned errors (MUE). The MSE is the mean of the algebraic differences, in degrees, between the rod adjustment and the gravita- 
tional vertical calculated over the eight trials. This measure estimates the accuracy of the eventual left/right deviation of the SV relative to the physical vertical. The MUE is the mean of the absolute differences, in degrees, between the rod adjustment and the gravitational vertical calculated over the eight trials. This measure estimates the precision of the adjustments. The results relative to these measures are summarized in Table 1, but the statistical analysis, which allowed us to test the haptic SV stability assumption, was performed on the MSE only, because it is this value that is kept, for each subject, in the explorationreproduction task. The .05 alpha level was adopted throughout. Post hoc analyses (comparison of each pair of means) were performed by Newman-Keuls test.

Analyses of variance (MSE). A 3 (body orientation) $\times 2$ (phase) analysis of variance (ANOVA), with repeated measures on these factors, revealed a significant effect of body orientation $[F(2,14)=11.529]$. As is depicted in Figure 1, an analysis confirmed the significance of the deviation of the haptic SV in the direction opposite to that of the body (E-effect) in the body-tilted conditions. In particular, post hoc analysis showed that the UB condition $\left(m=0.057^{\circ}\right)$ was significantly different from the BL condition $\left(m=7.592^{\circ}\right)$ and from the BR condition $\left(m=-7.559^{\circ}\right)$. The ANOVA did not reveal a significant effect of phase factor $[F(1,7)=1.686 ; p=$ .24] or an interaction between this factor and body orientation $[F(2,14)<1]$.

Analysis of correlations (MSE). The analysis of correlations (Bravais-Pearson coefficient) on the MSE showed that the haptic SV estimated in Phase 1 was positively correlated with the haptic SV estimated in Phase 2. This strong link was observed whatever the position of the body during the adjustments (for the UB condition, $r=$ .70 ; for the BL condition, $r=.92$; and for the BR condition, $r=.94)$.

\section{Exploration-Reproduction Task: The Haptic Oblique Effect}

In the exploration-reproduction task, the algebraic deviation between the standard rod orientation and the response rod orientation was noted for each trial. Deviations to the left of the standard orientation (rod turned counterclockwise) were counted as negative, and deviations to the right were counted as positive. In the case of a systematic bias concerning the oblique, an asymmetric effect was expected, since an overestimation of the angle between the $-45^{\circ}$ oblique orientation and the vertical would lead to negative deviations, whereas an overestimation between the $+45^{\circ}$ and the vertical would lead to positive deviations. The MUE and the MSE were then computed over the eight trials, for each of the five orientations tested, and for each subject. The results are summarized in Table 2 .

Mean unsigned errors. In order to know whether the classical haptic oblique effect (higher precision in vertical and horizontal orientations than in oblique orientations) was affected by body orientation, we first analyzed the MUE. A 3 (body orientation) $\times 5$ (rod orientation) ANOVA, with repeated measures on these factors, did not reveal a significant effect of body orientation $[F(2,14)=$ $3.244, p=.07]$. The rod orientation factor had an effect $[F(4,28)=8.944]$. A post hoc analysis showed that the SV $\left(m=2.622^{\circ}\right)$ was reproduced more precisely than the $0^{\circ}\left(m=4.280^{\circ}\right), 90^{\circ}\left(m=3.769^{\circ}\right),-45^{\circ}(m=$ $\left.5.475^{\circ}\right)$, and $+45^{\circ}\left(m=5.306^{\circ}\right)$ orientations. The body orientation $\times$ rod orientation interaction was also significant $[F(8,56)=3.200$; see Figure 2].

In order to test our hypotheses, a set of a priori orthogonal contrasts was tested. It showed that in the UB condition, the oblique effect $\left(0^{\circ}\right.$ and $90^{\circ}$ vs. $-45^{\circ}$ and $\left.+45^{\circ}\right)$ was significant $[F(1,7)=35.475]$. By contrast, in the two body-tilted conditions, this oblique effect was not significant $[F(1,7)<1]$. To investigate the role of the SV in body-tilted positions, we compared the SV orientation performances with the performances obtained in all the other orientations (SV vs. $0^{\circ}, 90^{\circ},-45^{\circ}$, and $+45^{\circ}$ ). The results showed that the $\mathrm{SV}$ orientation was reproduced more accurately than the other orientations in the BL condition $[F(1,7)=27.862]$, in the BR condition $[F(1,7)=$ $26.423]$, and even in the UB condition $[F(1,7)=22.747$; an $S V$ effect]. Moreover, a post hoc analysis did not show, for the upright posture, significant differences between: (1) $0^{\circ}$ and $90^{\circ}$ orientations $(p=.84),(2)-45^{\circ}$ and $+45^{\circ}$ orientations $(p=.81)$, and (3) $\mathrm{SV}$ and $0^{\circ}$ orientation $(p=.77)$.

Mean signed errors. In order to investigate the eventual existence of systematic deviations from the standard orientations, an analysis was conducted on the MSE. A 3 (body orientation) $\times 5$ (rod orientation) ANOVA, with repeated measures on these factors, revealed a main effect of body orientation $[F(2,14)=9.550]$. A post hoc test showed that the UB condition $\left(m=0.218^{\circ}\right)$ differed

Table 1

Experiment 1 (Production Task): The Haptic Subjective Vertical Estimated in Phase 1 (Before the Exploration-Reproduction Task) and Phase 2 (After the Exploration-Reproduction Task) as a Function of Body Position

\begin{tabular}{|c|c|c|c|c|c|c|c|c|c|c|c|c|}
\hline \multirow[b]{4}{*}{ Measure } & \multicolumn{12}{|c|}{ Postural Conditions } \\
\hline & \multicolumn{4}{|c|}{ Upright Body } & \multicolumn{4}{|c|}{ Body Tilted to the Left } & \multicolumn{4}{|c|}{ Body Tilted to the Right } \\
\hline & \multicolumn{2}{|c|}{ Phase 1} & \multicolumn{2}{|c|}{ Phase 2} & \multicolumn{2}{|c|}{ Phase 1} & \multicolumn{2}{|c|}{ Phase 2} & \multicolumn{2}{|c|}{ Phase 1} & \multicolumn{2}{|c|}{ Phase 2} \\
\hline & $M$ & $S D$ & $M$ & $S D$ & $M$ & $S D$ & $M$ & $S D$ & $M$ & $S D$ & $M$ & $S D$ \\
\hline MSE & 0.707 & 1.560 & -0.594 & 1.885 & 7.481 & 8.006 & 7.703 & 7.198 & -7.289 & 5.956 & -7.828 & 6.640 \\
\hline MUE & 1.746 & 0.801 & 1.734 & 1.093 & 8.246 & 7.305 & 8.320 & 6.553 & 7.867 & 5.261 & 8.484 & 6.199 \\
\hline
\end{tabular}

Note-MSE, mean signed errors; MUE, mean unsigned errors. These measures are expressed in degrees. 


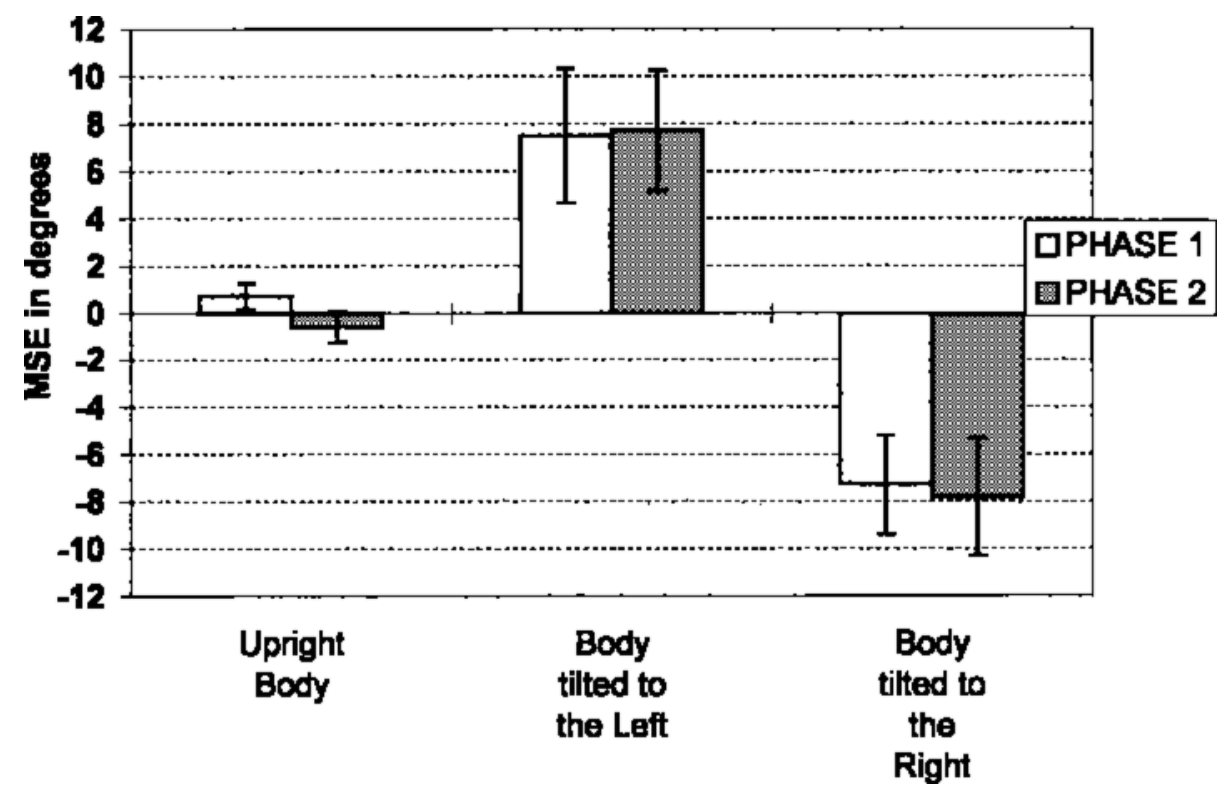

Figure 1. Experiment 1 (production task): The haptic subjective vertical stability as a function of the postural conditions and the phases of the experiment. MSE, mean signed errors, in degrees. Bars represent the standard error of the mean.

from the BL condition $\left(m=-2.163^{\circ}\right)$, but not from the BR condition $\left(m=1.505^{\circ}, p=.15\right)$. The ANOVA failed to show a significant effect of rod orientation $[F(4,28)=$ $1.635, p=.19$ ] or an interaction between the two factors $[F(8,56)<1$; see Figure 3].

\section{Production Task}

\section{Discussion}

The results obtained in the production task (Phases 1 and 2) showed systematic deviations of the haptic SV in a direction opposite to that of the body-in other words, a significant E-effect. This result confirms previous experiments (Bauermeister et al., 1964; Guerraz et al., in press). The comparison between Phases 1 and 2 showed that the perception of the gravitational vertical in the haptic modality during body tilt-in particular, the E-effect observed in this condition-did not change as a function of test time. Moreover, the high positive correlations found between postural conditions suggest that the haptic SV is also reliable over time, whatever the postural conditions. Therefore, despite the free hand movements during scanning, the haptic SV is a stable measure allowing us to examine the reproduction of this subjective orientation in the exploration-reproduction task.

\section{Exploration-Reproduction Task}

The results showed that body tilt had a disturbing effect on the precision of orientation reproduction(MUE) in particular, on the reproduction of the gravitational vertical and horizontal orientations. As a result, the haptic oblique effect found in the upright posture disappeared when the body was tilted. Thus, body tilts had an effect not only on the perception of the vertical (production task), but also on the reproduction of orientations (exploration-

Table 2

Experiment 1 (Reproduction Task): The Reproduction of Orientations as a Function of Body Position and the Value of Orientations

\begin{tabular}{|c|c|c|c|c|c|c|c|c|c|c|c|}
\hline \multirow[b]{3}{*}{ Measure } & \multirow[b]{3}{*}{ Posture } & \multicolumn{10}{|c|}{ Standard Orientations } \\
\hline & & \multicolumn{2}{|c|}{$0^{\circ}$} & \multicolumn{2}{|c|}{$90^{\circ}$} & \multicolumn{2}{|c|}{$-45^{\circ}$} & \multicolumn{2}{|c|}{$+45^{\circ}$} & \multicolumn{2}{|c|}{ SV } \\
\hline & & $M$ & $S D$ & $M$ & $S D$ & $M$ & $S D$ & $M$ & $S D$ & $M$ & $S D$ \\
\hline \multirow[t]{3}{*}{ MUE } & upright body & 2.449 & 0.638 & 2.305 & 0.993 & 6.059 & 1.994 & 5.180 & 1.659 & 1.970 & $\overline{0.462}$ \\
\hline & body to the left & 5.648 & 3.212 & 4.340 & 2.183 & 5.563 & 2.435 & 5.488 & 2.074 & 3.254 & 1.472 \\
\hline & body to the right & 4.742 & 0.754 & 4.664 & 1.835 & 4.805 & 1.814 & 5.250 & 1.712 & 2.644 & 0.917 \\
\hline \multirow[t]{3}{*}{ MSE } & upright body & 0.262 & 1.989 & 0.586 & 0.918 & -0.61 & 4.423 & 1.633 & 2.466 & -0.777 & 0.981 \\
\hline & body to the left & -2.789 & 4.819 & -.1 .535 & 3.683 & -3.734 & 3.141 & -1.324 & 2.367 & -1.434 & 1.824 \\
\hline & body to the right & 1.914 & 3.055 & 1.867 & 3.921 & 0.289 & 3.674 & 2.984 & 2.446 & 0.473 & 1.550 \\
\hline
\end{tabular}

Note-SV, Subjective vertical; MUE, mean unsigned errors; MSE, mean signed errors. Measures are expressed in degrees. 


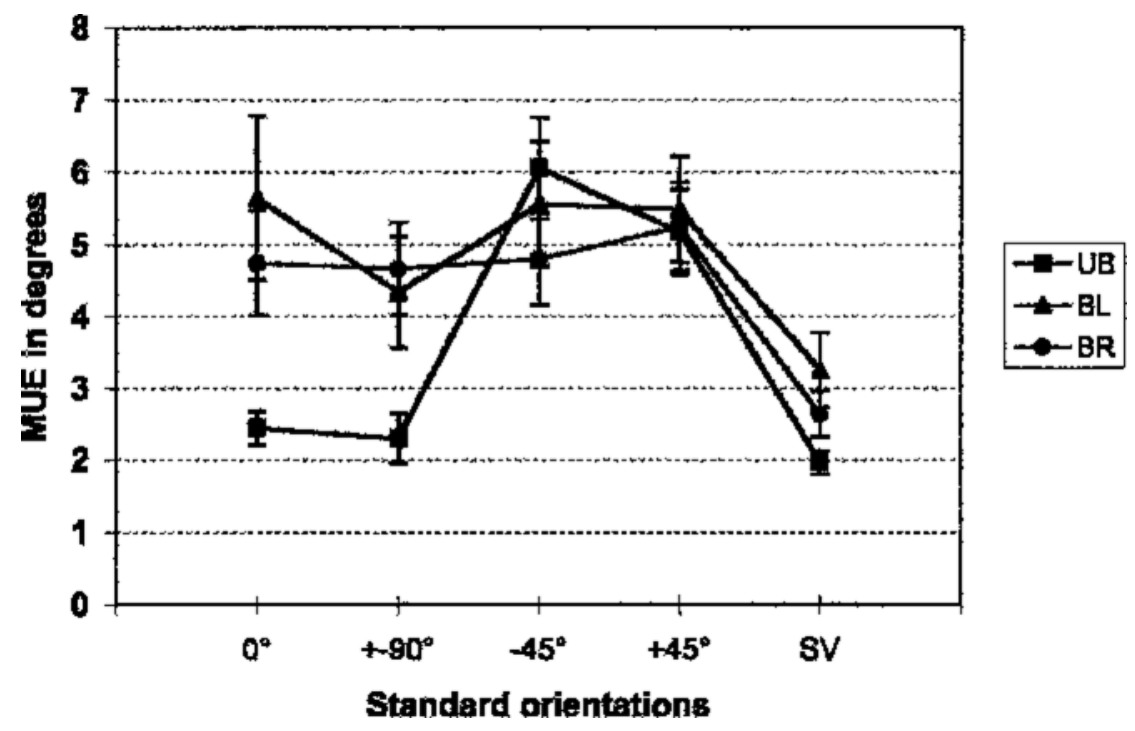

Figure 2. Experiment 1 (reproduction task): The reproduction of orientations as a function of body position. UB, upright body; BL, body tilted to the left; BR, body tilted to the right; SV, subjective vertical; MUE, mean unsigned errors, in degrees. Bars represent the standard error of the mean.

reproduction task). This means that, in tilted postures, gravitational vertical and horizontal orientations seemed no longer to have the role of reference norms, since they were not reproduced better than the $45^{\circ}$ oblique orientations. Therefore, the hypothesis of a gravitational reference frame underlying the oblique effect is not supported. Similarly, the results do not favor an egocentric reference frame, since the oblique orientations that were vertical and horizontal by reference to the body axis in a tilted posture were not perceived more accurately than the physical vertical and horizontal orientations. Actually, the results showed that the SV orientation was reproduced more accurately than the other orientations-in particular, in the body-tilted conditions. In these conditions, the SV could constitute a norm axis for a subjective gravitational reference frame that could be especially important when the body is tilted.

The analysis of the MSE showed that body tilts tended to increase reproduction errors in the same direction of the body whatever the value of standard orientations. It

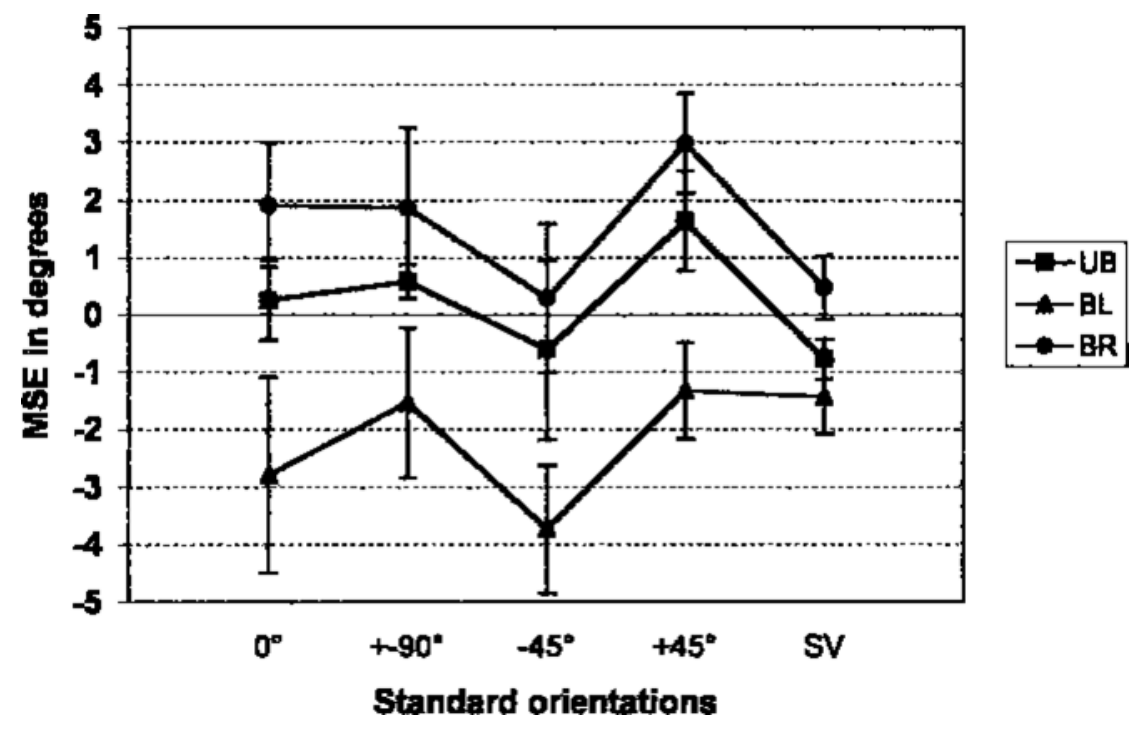

Figure 3. Experiment 1 (reproduction task): the reproduction of orientations as a function of body position. UB, upright body; BL, body tilted to the left; BR, body tilted to the right; SV, subjective vertical; MSE, mean signed errors, in degrees. Bars represent the standard error of the mean. 
should be noted that the reverse effect was obtained in the production task when the subject had to adjust the rod to the gravitational vertical: The SV deviated in a direction opposite to that of the body because the rod set to the physical vertical appeared to be tilted toward the body (apparent inclination). Thus, when the subject has to reproduce orientations, body tilts seem to increase this apparent inclination of the rod, since if the apparent inclination were strictly reproduced, no error should be observed. Further studies are necessary to elucidate the origin of this effect.

However, this experiment raises several questions. First, when the whole body was tilted, the arm-handdigit system was rotated, and scanning movements of the shoulder-hand system in this condition were not strictly comparable with those permitted in the upright posture. Moreover, different hands were used in each condition: the left hand in the BR condition and the right hand in the BL condition. This factor should be controlled, although Bauermeister et al.'s (1964) experiment tended to show that this procedure (left hand for BR and right hand for $\mathrm{BL})$ gave rise to comparable deviations in a production task. A second question concerns the role of the vestibular system, as compared with the somesthetic system, in the body tilt effect observed in the reproduction of orientations. When the whole body is tilted, several kinds of proprioceptors normally code the body tilt: the otolith organs in the vestibular system; the cutaneous receptors activated by the change in the distribution of pressure on the skin; the muscular tension, which becomes asymmetrical with respect to the gravitational vertical; and the graviceptive receptors in the trunk, located in the kidneys and the vascular system (Mittelstaedt, 1997). It would therefore be interesting to restrict the tilt to the head in order to isolate only the information provided by the vestibular system and the neck receptors.

\section{EXPERIMENT 2 \\ Head Tilt Effects on the Haptic Oblique Effect}

The main goal of this experiment was to test the tilt effect of the head alone on the reproduction of orientations. Tilting only the head, by contrast to whole-body tilts, allows us to keep the arm-hand-digit system relatively independent from the disturbance caused by the tilt. The same hand (dominant hand) can be used what- ever the direction of tilt (left vs. right), and this head tilt prevents the strong asymmetrical somesthetic stimulation of the body below the neck. Thus, head tilt activates mainly the otolith organs, the neck muscles, and the neck joint receptors. Moreover, contrary to what occurs in the whole-body tilt, the trunk remains vertical during head tilt, and as was suggested by Chen and Levi (1996), it could be used as a reference axis in line with a gravitational reference frame.

\section{Method}

\section{Subjects}

The subjects were 8 right-handed students in psychology ( 4 females, 4 males; mean age, 25.75 years). Their handedness was assessed by Bryden's (1977) five-item hand preference questionnaire. On the basis of self-report, the subjects had no hearing or vestibular disorders, including diseases with ocular manifestation or motion sickness.

\section{Apparatus}

The haptic adjustments were made with the same apparatus as that depicted in Experiment 1. The subjects sat on a chair with an adjustable headrest that could be moved along the tilt axis to accommodate different body sizes. The headrest was padded, could be rotated to $45^{\circ}$ left or right, and was aligned with the physical vertical.

\section{Experimental Conditions and Procedure}

The subject wore an occluded dark mask on the eyes during the experiment. He or she was sitting. The procedure was the same as that in Experiment 1: It comprised three sessions corresponding to three postural conditions: head to the vertical (HV), head tilted to the left (HL), and head tilted to the right (HR). There was about 1 week between the different sessions, and their order was randomized. In each session, each subject was tested in three successive periods: (1) a production task (Phase 1), (2) an exploration-reproduction task, and (3) a production task (Phase 2). There was a delay of about 5 min between the three periods. As in Experiment 1, a session comprised 56 test trials- $(1$ [orientation $) * 2$ [Phase 1 and Phase 2] $* 8$ trials $)+(8$ trials $* 5$ standard orientations $)$ - for a total of 168 test trials per subject. The same hand (the right) was used in all the conditions. As a consequence, for the reproduction of the horizontal orientation, only the $-90^{\circ}$ orientation was tested.

\section{Results}

\section{Production Task}

The results are summarized in Table 3 .

Analyses of variance (MSE). A 3 (head orientation) $\times 2$ (phase) ANOVA on MSE, with repeated measures

Table 3

Experiment 2 (Production Task): The Haptic Subjective Vertical Estimated

in Phase 1( Before the Exploration-Reproduction Task) and Phase 2 (After the Exploration-Reproduction Task) as a Function of Head Position

\begin{tabular}{|c|c|c|c|c|c|c|c|c|c|c|c|c|}
\hline \multirow[b]{4}{*}{ Measure } & \multicolumn{12}{|c|}{ Head Orientation } \\
\hline & \multicolumn{4}{|c|}{ Head to the Vertical } & \multicolumn{4}{|c|}{ Head to the Left } & \multicolumn{4}{|c|}{ Head to the Right } \\
\hline & \multicolumn{2}{|c|}{ Phase 1} & \multicolumn{2}{|c|}{ Phase 2} & \multicolumn{2}{|c|}{ Phase 1} & \multicolumn{2}{|c|}{ Phase 2} & \multicolumn{2}{|c|}{ Phase 1} & \multicolumn{2}{|c|}{ Phase 2} \\
\hline & $M$ & $S D$ & $M$ & $S D$ & $M$ & $S D$ & $M$ & $S D$ & $M$ & $S D$ & $M$ & $S D$ \\
\hline MSE & 0.387 & 1.623 & -0.371 & 1.593 & 6.395 & 4.183 & 7.656 & 5.846 & -7.109 & 2.617 & -8.106 & 3.786 \\
\hline MUE & 1.957 & 0.947 & 1.848 & 0.724 & 7.105 & 3.176 & 8.320 & 4.718 & 7.109 & 2.617 & 8.184 & 3.657 \\
\hline
\end{tabular}

Note-MSE, mean signed errors; MUE, mean unsigned errors. These measures are expressed in degrees. 


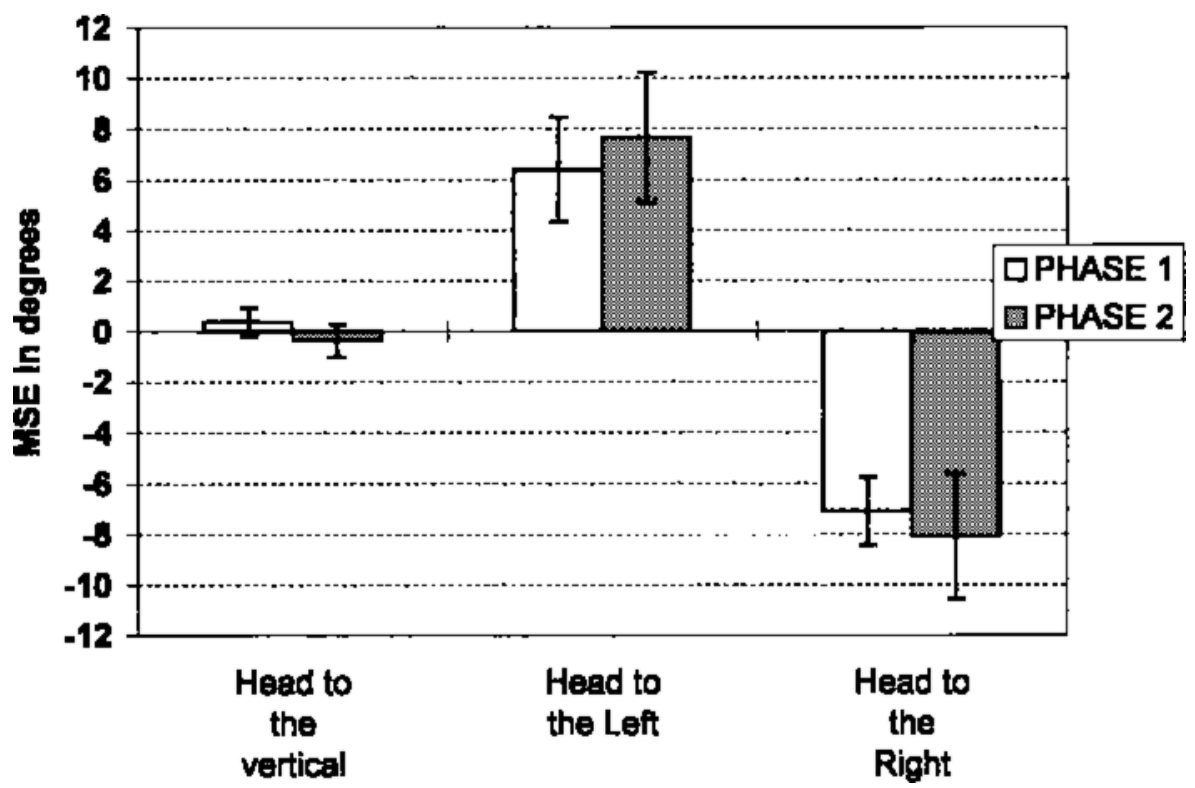

Figure 4. Experiment 2 (production task): The perception of orientations as a function of head position and the phases of the experiment. MSE, mean signed errors, in degrees. Bars represent the standard error of the mean.

on these factors, revealed a significant effect of head orientation $[F(2,14)=38.419]$. As is depicted in Figure 4, the analysis confirmed the significance of the deviation of the haptic SV in the direction opposite to that of the head (E-effect) in head-tilted conditions. A post hoc analysis showed that the $\mathrm{HV}$ condition $\left(m=0.007^{\circ}\right)$ was significantly different from the HL condition $\left(m=7.025^{\circ}\right)$ and from the HR condition $\left(m=-7.607^{\circ}\right)$. The ANOVA did not reveal a significant effect of phase factor $[F(1,7)$ $<1]$ or an interaction between this factor and body orientation $[F(2,14)=1.051, p=.38]$.

Analysis of correlations (MSE). The analysis of correlations (Bravais-Pearson coefficient) on MSE showed that the haptic SV estimated in Phase 1 was positively and significantly correlated with the haptic SV estimated in Phase 2 for the HL condition only $(r=.92)$. The analysis failed to show a significant link between Phases 1 and 2 in the HR condition $(r=.25)$ or between Phases 1 and 2 in the HV condition $(r=-.44)$.

\section{Exploration-Reproduction Task}

The results of the exploration-reproduction task are summarized in Table 4.

Mean unsigned errors. A 3 (head orientation) $\times 5$ (rod orientation) ANOVA, with repeated measures on these factors, on the MUE failed to show a significant effect of the head position factor $[F(2,14)=2.814, p=$ $.09]$. However, the analysis showed a significant effect of orientations $[F(4,28)=4.756]$. A post hoc analysis revealed that the $\mathrm{SV}\left(m=2.538^{\circ}\right)$ was reproduced more accurately than the horizontal orientation $\left(m=3.795^{\circ}\right)$, the $-45^{\circ}$ orientation $\left(m=4.509^{\circ}\right)$, and the $+45^{\circ}(m=$

Table 4

Experiment 2 (Reproduction Task): The Reproduction of Orientations as a Function of Head Orientation and the Value of Orientations

\begin{tabular}{|c|c|c|c|c|c|c|c|c|c|c|c|}
\hline \multirow[b]{3}{*}{ Measure } & \multirow[b]{3}{*}{ Head Orientation } & \multicolumn{10}{|c|}{ Standard Orientations } \\
\hline & & \multicolumn{2}{|c|}{$0^{\circ}$} & \multicolumn{2}{|c|}{$90^{\circ}$} & \multicolumn{2}{|c|}{$-45^{\circ}$} & \multicolumn{2}{|c|}{$+45^{\circ}$} & \multicolumn{2}{|c|}{ SV } \\
\hline & & $M$ & $S D$ & $M$ & $S D$ & $M$ & $S D$ & $M$ & $S D$ & $M$ & $S D$ \\
\hline \multirow[t]{3}{*}{ MUE } & head to the vertical & 2.019 & 0.744 & 2.797 & 0.804 & 5.219 & 2.104 & 4.219 & 1.713 & 1.813 & 0.357 \\
\hline & head to the left & 4.480 & 2.780 & 4.664 & 1.951 & 4.223 & 1.574 & 3.703 & 1.270 & 1.997 & 1.870 \\
\hline & head to the right & 3.719 & 0.930 & 3.926 & 1.457 & 4.086 & 1.310 & 4.191 & 0.732 & 2.772 & 1.132 \\
\hline \multirow[t]{3}{*}{ MSE } & head to the vertical & -0.449 & 1.330 & -0.375 & 1.610 & -2.016 & 2.829 & 1.781 & 1.665 & -0.891 & 0.304 \\
\hline & head to the left & -3.113 & 3.641 & -0.648 & 3.846 & -0.980 & 2.788 & -0.070 & 1.662 & -1.188 & 3.058 \\
\hline & head to the right & -0.172 & 1.810 & 1.332 & 2.292 & -1.820 & 2.524 & 2.270 & 1.856 & 1.277 & 2.289 \\
\hline
\end{tabular}

Note-SV, subjective vertical; MUE, mean unsigned errors; MSE, mean signed errors; Measures are expressed in degrees. 


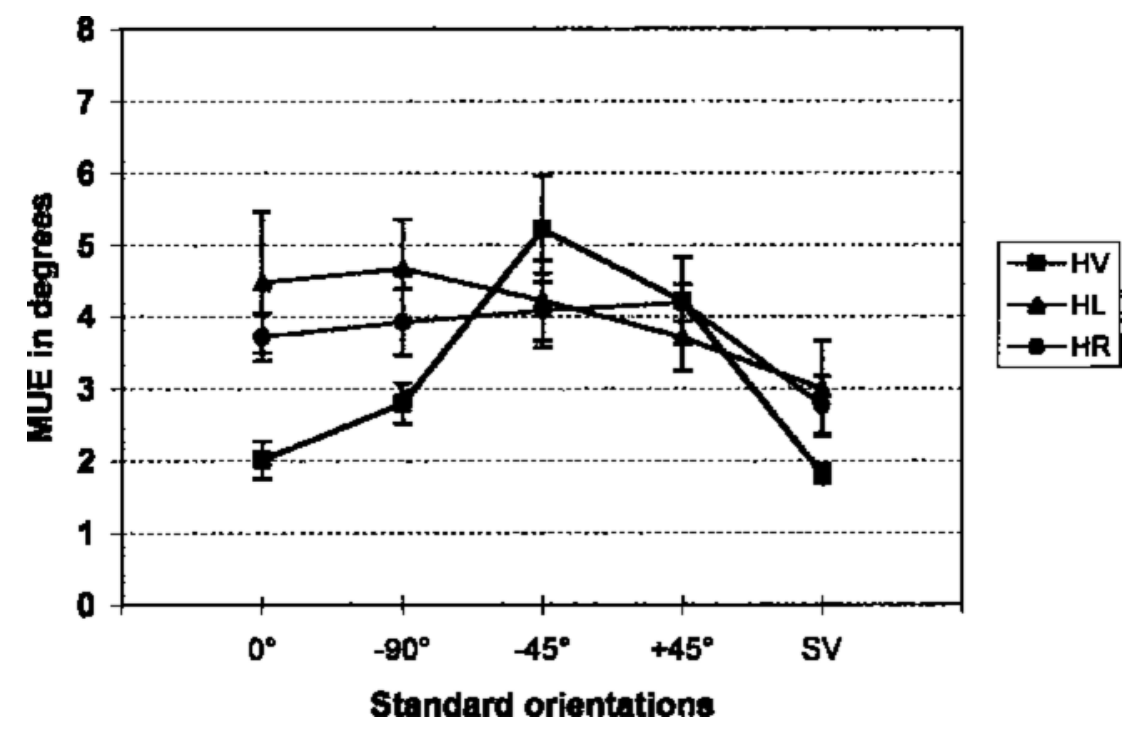

Figure 5. Experiment 2 (reproduction task): The reproduction of orientations as a function of head position. HV, head in the vertical position; HL, head tilted to the left; HR, head tilted to the right; SV, subjective vertical; MUE, mean unsigned error, in degrees. Bars represent the standard error of the mean.

$4.038^{\circ}$ ). Only a tendency was observed between the SV and the vertical orientation $(m=3.404, p=.08)$. The interaction between the two factors was significant $[F(8,56)=3.785$; see Figure 5].

A set of a priori orthogonal contrasts was computed in order to test our hypotheses. This analysis showed that in the $\mathrm{VH}$ condition, the classical oblique effect was significant $[F(1,7)=18.354]$. This effect was no longer present when the head was tilted $[F(1,7)<1$ for both the HL and HR conditions]. By contrast, an SV appeared when the SV was compared with all other orientations in the tilted conditions $[F(1,7)=6.829$ for the HL condition and $F(1,7)=20.660$ for the HR condition].

Moreover, a post hoc analysis did not show, for the upright posture, a difference between $0^{\circ}$ and $-90^{\circ}$ orientations $(p=.37)$ or between $-45^{\circ}$ and $+45^{\circ}$ orientations $(p=.42)$. The difference between the $\mathrm{SV}$ and the $0^{\circ}$ orientation was not significant ( $p=.73$ ) in the HV condition. The differences between the $-45^{\circ}$ orientation in the HV condition and the $-45^{\circ}$ orientation in the HL and HR conditions (see Figure 5) were not significant ( $p=.31$ and .45 , respectively).

Mean signed errors. In order to investigate the presence of systematic deviations in the final adjustments, an analysis was conducted on the MSE (see Figure 6). A 3 (head orientation) $\times 5$ (rod orientation) ANOVA, with repeated measures on these factors, revealed a main effect of body orientation $[F(2,14)=3.786]$. A post hoc test failed to reveal a difference between the HV condition $\left(m=-0.390^{\circ}\right)$ and the HL condition $(m=$ $\left.-1.200^{\circ}, p=.23\right)$ or between the HV condition and the HR condition $\left(m=0.577^{\circ}, p=.16\right)$. However, the HL and HR conditions were significantly different: Tilting the head to the left or to the right led to deviations in the direction of the head.

The ANOVA also showed an effect of rod orientations $[F(4,28)=6.588]$. The reproduction of the vertical $(m=$ $\left.-1.245^{\circ}\right)$, the $-45^{\circ}\left(m=-1.605^{\circ}\right)$, and the SV $(m=$ $-0.267^{\circ}$ ) orientations led to negative deviations, whereas the reproduction of the horizontal and the $+45^{\circ}$ orientations led to positive deviations. The difference between the $-45^{\circ}$ and the $+45^{\circ}$ orientations was significant, which means that there was a tendency to overestimate the angle between the vertical and these oblique orientations. The difference between the reproduction of the $0^{\circ}$ orientation and that of the $+45^{\circ}$ orientation was also significant. The $\mathrm{SV}$ orientation differed from the $+45^{\circ}$ orientation, but all other comparisons of pairs of means were not significant. Moreover, the ANOVA failed to reveal a significant effect of the interaction between the two factors $[F(8,56)=$ $1.244, p=.29]$.

\section{Discussion}

The results found in the production task have shown that, similar to Experiment 1 when the whole body was tilted, tilting only the head led to systematic deviations in the direction opposite to that of the head (E-effect). The comparison between Phase 1 and Phase 2 has shown a very good stability of the haptic SV during the experiment. However, for unexplained reasons, the reliability was not as good as that found in Experiment 1.

The exploration-reproduction task showed that tilting only the head produced the same effect on the reproduction of orientations as tilting the whole body (Experiment 1). The principal orientations (vertical and horizontal) were affected by head position and were not 


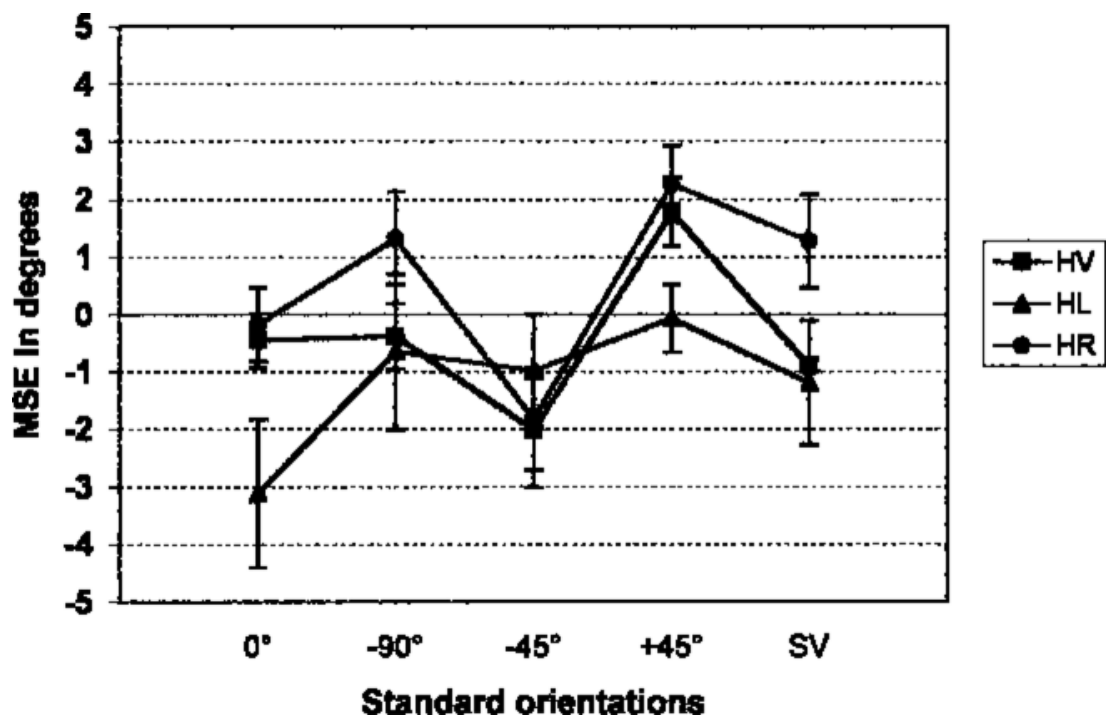

Figure 6. Experiment 2 (reproduction task): The reproduction of orientations as a function of head position. HV, head in vertical position; HL, head tilted to the left; HR, head tilted to the right, SV, subjective vertical; MSE, mean signed errors, in degrees. Bars represent the standard error of the mean.

reproduced better than the oblique orientations (no oblique effect) in the tilted-head postures.

\section{EXPERIMENT 3 \\ Head Tilt Effects on Subjective Orientations}

The aim of Experiment 3 was to investigate the head tilt effects on the production of different orientations in the haptic modality (and not only the production of the physical vertical, as in Experiments 1 and 2). Thus, four different orientations were tested: $0^{\circ}(\mathrm{SV}), 90^{\circ}$ (subjective horizontal, or $\mathrm{SH}$ ), $-45^{\circ}$, and $+45^{\circ}$ (subjective obliques). The question was whether the systematic bias in the direction opposite to the body or the head (E-effect) found in Phase 1 of Experiments 1 and 2 was restricted to the vertical orientation or whether other orientations were also influenced by head tilt.

Although the perception of the gravitational vertical and horizontal have been extensively investigated in the visual modality (with rod adjustments), very few data (in particular, see Kerkhoff, 1999; Kerkhoff \& Zoelch, 1998) are available on oblique axes. Moreover, to our knowledge, adjustments made by subjects to oblique axes without standard orientations being visible during the adjustment, and the effect of body tilt on this oblique perception have not yet been studied in the haptic modality.

\section{Method}

\section{Subjects}

The subjects were 16 right-handed students in psychology (11 females, 5 males; mean age, 24 years). Their handedness was assessed by Bryden's (1977) five-item hand preference questionnaire. On the basis of self-report, the subjects had no hearing or vestibular disorders, including diseases with ocular manifestation or motion sickness.

\section{Apparatus}

The haptic adjustments were made with the same apparatus as that depicted in Experiment 2.

\section{Experimental Conditions and Procedure}

The subjects were asked to align the haptic rod to four different orientations: $0^{\circ}(\mathrm{SV}), 90^{\circ}(\mathrm{SH}),-45^{\circ}$, and $+45^{\circ}$. The same gravitational definition as that in Experiments 1 and 2 (Phases 1 and 2) was given for the physical vertical. The horizontal was defined as being perpendicular to the physical vertical, and the subject was free to choose the $-90^{\circ}$ or the $+90^{\circ}$ orientation. Oblique orientations were defined as being the middle of the right angle constituted by the vertical and the horizontal. Four trials were performed for each orientation, and the order of the different orientations was randomized. The subjects performed this task in three head posture conditions: $\mathrm{HV}$, HL $\left(-45^{\circ}\right)$, and $\mathrm{HR}\left(+45^{\circ}\right)$. Therefore, the production task comprised 48 test trials $(3$ head positions $\times 4$ orientations $\times$ 4 trials). For each trial, the algebraic deviation from the tested orientations, in degrees, was noted. Deviations to the left (rod turned counterclockwise) were counted as negative, and deviations to the right (clockwise) were counted as positive.

\section{Results}

In order to test whether the E-effect (deviations opposite to that of the head) was present whatever the value of orientations, an analysis was conducted only on the MSE. A 3 (head orientation $) \times 4$ (rod orientation $)$ ANOVA, with repeated measures on these factors, revealed a main effect of head orientation $[F(2,30)=62.291]$. A systematic deviation in the direction opposite to the head appeared in tilted positions $\left(m=6.016^{\circ}\right.$ for the HL condition, and $m=-3.272^{\circ}$ for the HR condition) relative to the upright head position $\left(m=0.812^{\circ}\right)$. The analysis failed to show a significant effect of the rod orientation factor $[F(3,45)<1]$ or an interaction between the two factors $[F(6,90)<1$; see Figure 7$]$. 


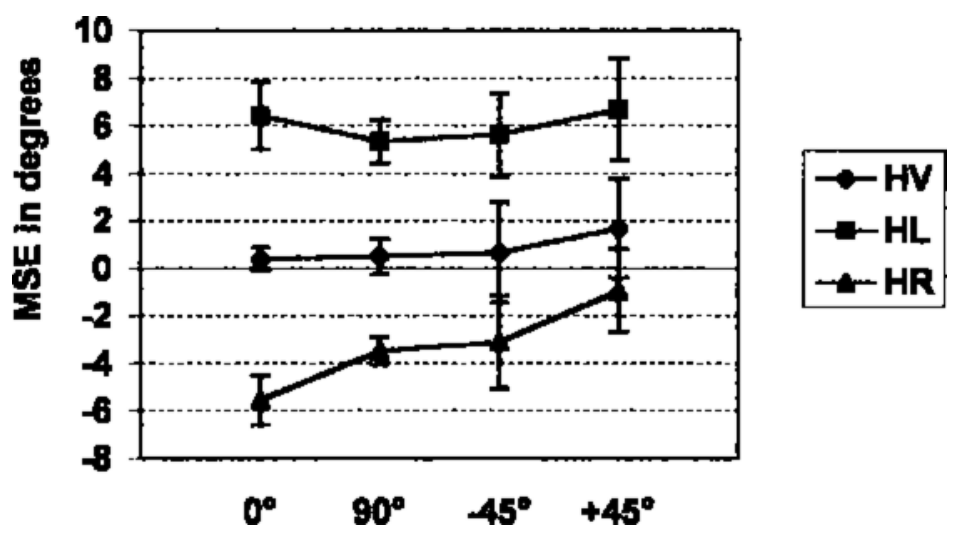

Figure 7. Experiment 3:The perception of orientations (MSE: mean signed errors in degrees, with error bars $\pm S E M$ ) as a function of head position. $\mathrm{HV}$, head in vertical position; HL, head tilted to the left; HR, head tilted to the right; MSE, mean signed error, in degrees. Bars represent the standard error of the mean.

\section{GENERAL DISCUSSION}

In this research, we have studied the effects of wholebody tilt (Experiment 1) and head tilt alone (Experiment 2) on the haptic oblique effect in order to determine the spatial reference frame in which orientations were defined. Body tilt and head tilt uncouple the egocentric and gravitational axes and specify the nature of the reference frame (egocentric vs. gravitational) involved in the oblique effect found previously in haptics tasks involving the exploration-reproduction of orientation. We studied the possible involvement of another reference frame, the subjective gravitational. In a production task, we measured the apparent deviation of the perception of the gravitational vertical-that is, the SV (Experiments 1 and 2, Phases 1 and 2). We further tested the reproduction of the SV in each subject. In addition, in Experiments 1 and 2, the stability and reliability of the haptic SV were assessed, because a previous report had suggested that free scanning of the rod during haptic adjustments led to a high variability of measures. Finally, in Experiment 3 , the effect of head tilt on the production of several haptic orientations (vertical, horizontal, and obliques) was also investigated. In particular, the problem was to know whether the deviation of the SV in a direction opposite to the head (E-effect) was restricted to the vertical orientation (as had been found in previous studies) or whether other orientations were also affected by head tilt. In this perspective, a general head tilt effect on all orientations would be another argument in favor of the existence of a subjective gravitational reference frame.

\section{Exploration-Reproduction Task}

We shall examine first the body and head tilt effects on the haptic oblique effect tested by the explorationreproduction task. Experiment 1 revealed a clear effect of body position on the haptic reproduction of orientations, and a similar effect was obtained when only the head was tilted (Experiment 2). The classical oblique ef- fect, observed in standing and sitting upright postures, is consistent with previous studies (Gentaz \& Hatwell, $1995,1996,1998)$. However, this anisotropy disappeared when the whole body or the head alone was tilted in the roll plane. Actually, body or head tilt decreased the precision of vertical and horizontal settings. As a result, the performances obtained in these dominant orientations were at the same poor level as those obtained in oblique ones. Therefore, the results of the two experiments did not support either a pure gravitational or an egocentric reference frame hypothesis but tended to support a "subjective" gravitational reference frame hypothesis.

Indeed, the main result was that the haptic oblique effect was present in tilted postures when all other orientations were compared with the haptic SV. This means that the haptic SV is an important axis, especially in tilted postures, and could constitute a reference norm. This result is not consistent with the apparent gravitational reference frame found in Van Hof and Lagers-van Haselen's (1994) experiment testing the proprioceptive encoding of parallelism. But in this latter study, the method used asked the subject to decide whether two bars grasped simultaneously, one by each hand, were parallel or not. This task was very different from the method used here, which consisted in the encoding and then the reproduction of a given orientation with free haptic exploration of the rod. The forced-choice method, in particular, may not distinguish between a subjective reference frame and a gravitational one, because the subjective frame is closer to the physical gravitational reference frame than to the egocentric one in the $45^{\circ}$ body tilt. An additional condition with bars oriented in the direction of the SV of each subject would be necessary to investigate the role of the subjective reference frame in the parallelism discrimination task of Van Hof and Lagers-van Haselen's experiment.

In the present research, the fact that tilting the head alone produced a similar effect as whole-body tilt suggests that the body tilt effect was not due to scanning movements of the shoulder-hand system (which was not the 
same, for a same orientation, in body-upright and bodytilted conditions) or to a difference in the hand used during adjustments in the two tilted conditions. It means, rather, that the position of the head is crucial not only for the perception of orientation (production task), as was found in previous studies (Guerraz, Poquin, Luyat, \& Ohlmann, 1998), but also for the reproduction of memorized orientations. The vestibular system, the joint, and the neck muscle receptors appeared, therefore, to play a dominant role, as compared with somesthetic cues provided by lower parts of the body (down from the neck).

Moreover, the body (and head) tilt effect on the reproduction of orientations is difficult to explain as being the result of a misperception of body position, since the subject's position did not change between the exploration of the standard rod and its reproduction and apparently no compensation of the body tilt was needed. This is very different from the production task, in which the system had to determine the inclination of the body in order to compensate for it in adjusting the rod to the physical vertical. The tendency of MSE to deviate systematically in the direction of the body or the head suggests that the subjective tilt of the rod during head or body tilt was enhanced (a physically vertical rod appeared tilted toward the head in these postures). An increase in the perceptual overestimation of the body position during the successive operations of the exploration-reproduction task (encoding, memorization, and reproduction) could be responsible for this increase in the apparent tilt of the rod, although previous reports have shown that body tilt effects on the SV (visual E-effect) tended to decrease with time (N. J. Wade, 1970). Rather, our results are in accordance with Mittelstaedt's (1983) model, in which the SV is computed from a gravity vector and an idiotropic vector, the idiotropic vector having a central origin. However, further experiments are necessary to investigate the eventual change in the perception of body position during the successive operations. Finally, the question remains of knowing at what level of the exploration-reproduction task (encoding, memorization, and reproduction) body and head tilt have an effect.

\section{Production Task}

We shall now discuss the effect of body and head tilt on the perception of haptic orientations, as estimated in the production task. The comparison between Phase 1 and Phase 2 (Experiment 1, in particular) has shown a good stability and reliability of the haptic SV. Contrary to a previous suggestion (S. Wade \& Curthoys, 1997), this finding suggests, therefore, that haptic adjustments implying free-scanning hand movements constitute a relatively satisfactory method of measurement. However, it would be interesting to compare different methods of adjustment in upright and tilted conditions-in particular, the proprioceptive coding of orientation and the haptic coding of orientation. These experiments could lead to a better understanding of the processes implied in these respective modalities, and they could determine the best method of estimation of the SV without vision.
The results of Experiment 3 (production task) showed that the E-effect produced by head tilt was not restricted to the haptic perception of the physical vertical but was also at work in the haptic perception of horizontal and oblique orientations. These findings are consistent with the idea that the SV and, to a minor extent, the SH constitute the reference norms of a subjective reference frame. During the head tilt in the roll plane, the entire subjective reference frame appears to be tilted the same amount in a direction opposite to that of the head.

Finally, given the apparent role played by subjective orientations in orientation processing, it would be interesting to test the effect of these subjective orientations in the visual modality. In visual adjustments, a strong link has been found between the deviation of the visual SV during illusory body tilts produced in a centrifuge and ocular countertorsion (S. Wade \& Curthoys, 1997). This could be related to the retinal coding of orientations obtained by Chen and Levi (1996) for the discrimination of parallelism when the ocular countertorsion of the eyes was controlled.

In summary, the present research showed that tilting the whole body or the head alone had a clear effect on the haptic reproduction of orientations. The classical oblique effect observed in the upright posture disappeared in tilted positions, mainly because of a decrease in the precision of the reproduction of vertical and horizontal orientations. In tilted positions, the SV (Experiments 1 and 2) appeared to be processed more accurately than all the other orientations. Thus, haptic orientations seemed to be mapped in neither a gravitational nor an egocentric reference frame but, rather, in a subjective gravitational reference frame.

\section{REFERENCES}

ApPelle, S. (1972). Perception and discrimination as a function of stimulus orientation: The "oblique effect" in man and animals. Psychological Bulletin, 78, 266-278.

Appelle, S., \& Countryman, M. (1986). Eliminating the haptic oblique effect: Influence of scanning incongruity and prior knowledge of the standards. Perception, 15, 365-369.

Attneave, F., \& Olson, R. K. (1967). Discriminability of stimuli varying in physical or retinal orientation. Journal of Experimental Psychology, 74, 149-157.

BAlliet, R, \& NAKAYAMA, K. (1978). Egocentric orientation is influenced by trained voluntary cyclorotary eye movements. Nature, $\mathbf{2 7 5}$, 214-216.

Banks, M. S., \& Stolarz, S. J. (1975). The effect of head tilt on meridional differences in acuity: Implications for orientation constancy. Perception \& Psychophysics, 17, 17-22.

Bauermeister, M., Werner, H., \& WAPner, S. (1964). The effect of body tilt on tactual-kinesthetic perception of verticality. American Journal of Psychology, 77, 451-456.

Bles, W., \& DE GRAAF, B. (1991). Ocular rotation and perception of the horizontal under static tilt conditions in patients without labyrinthine function. Acta Otolaryngologica, 111, 456-462.

BRY DEN, M. P. (1977). Measuring handedness with questionnaires. Neuropsychologia, 15, 617-624.

Buchanan-Smith, H. M., \& Heeley, D. W. (1993). Anisotropic axes in orientation perception are not retinotopically mapped. Perception, 22, 1389-1402.

Cecala, A. J., \& Garner, W. R. (1986). Internal frame of reference as a determinant of the oblique effect. Journal of Experimental Psychology: Human Perception \& Performance, 12, 314-323. 
Chen, S., \& Levi, D. M. (1996). Meridional anisotropy in the discrimination of parallel and perpendicular lines-effect of body tilt. Perception, 25, 633-649.

Corwin, T. R, Moskowitz-Cook, A., \& Green, M. A. (1977). The oblique effect in a vernier acuity situation. Perception \& Psychophysics, 21, 445-449.

DeFord, J. K., Prather, J. R., \& Essock, E. A. (1998). Effect of alternative frames of reference on the oblique effect of visual search in varied viewing positions [Abstract]. Investigative Ophthalmology \& Visual Science, 39 (Suppl.), S167.

DeFord, J. K., Prather, J. R., \& Essock, E. A. (1999).The effect of frame-of-reference and target knowledge on the oblique effect of visual search [Abstract]. Investigative Ophthalmology \& Visual Science, 40 (Suppl.), S347.

DE GRAAF, B. (1990). Influence of eye movements on visual perception. Unpublished doctoral dissertation, University of Utrecht.

Essock, E. A. (1980). The oblique effect of stimulus identification considered with respect to two classes of oblique effects. Perception, 9, 37-46.

Essock, E. A., Krebs, W. K., \& Prather, J. R. (1997). Superior sensitivity for tactile stimuli oriented proximally-distally on the finger: Implications for mixed class 1 and class 2 anisotropies. Journal of Experimental Psychology: Human Perception \& Performance, 23, 515-527.

Ferrante, D., Gerbino, W., \& Rock, I. (1995). Retinal vs. environmental orientation in the perception of the right angle. Acta Psychologica, 88, 25-32.

Furmanski, C., \& ENGel, A. (2000). An oblique effect in human primary visual cortex. Nature Neuroscience, 3, 535-536.

GentaZ, E. (2000). Existe-t-il un effet de l'oblique dans la perception tactile des orientations? [Is there an oblique effect in the tactual perception of orientations?] L'Année Psychologique, 100, 111-140.

Gentaz, E., \& Ballaz, C. (in press). La perception visuelle des orientations et l'effet de l'oblique. [The visual perception of orientations and the "oblique effect".] L'Année Psychologique.

Gentaz, E., \& Hatwell, Y. (1995). The haptic "oblique effect" in children's and adults' perception of orientation. Perception, 24, 631646.

Gentaz, E., \& Hatwell, Y. (1996). Role of gravitational cues in the haptic perception of orientation. Perception \& Psychophysics, 58, 1278-1292.

Gentaz, E., \& Hatwell, Y. (1998). The haptic oblique effect in the perception of rod orientation by blind adults. Perception \& Psychophysics, 60, 157-167.

Gentaz, E., \& Hatwell, Y. (1999). Role of memorisation conditions in the haptic processing of orientations and the "oblique effect." British Journal of Psychology, 90, 373-388.

Gentaz, E., Luyat, M., Cian, C., Hatwell, Y., Barraud, P.-A., \& RAPHEL, C. (in press). The reproduction of vertical and oblique orientations in the visual, haptic, and somato-vestibular systems. Quarterly Journal of Experimental Psychology.

Guerraz, M., Luyat, M., Poquin, D., \& Ohlmann, T. (in press). The role of neck afferents on subjective orientation in the visual and tactile sensorial modalities. Acta Otolaryngologica.

Guerraz, M., Poquin, D., Luyat, M., \& Ohlmann, T. (1998). Head orientation involvement in assessment of the subjective vertical during whole body tilt. Perceptual \& Motor Skills, 87, 643-648.
Heeley, D., \& Buchanan-Smith, H. (1990). Recognition of stimulus orientation. Vision Research, 30, 1429-1437.

Heeley, D., Buchanan-Smith, H., Cromwell, J., \& Wright, J. (1997). The oblique effect in orientation acuity. Vision Research, 32, 235-242.

Howard, I. P. (1982). Human visual orientation. New York: Wiley. KerKHOFF, G. (1999). Multimodal spatial orientation deficits in leftsided visual neglect. Neuropsychologia, 37, 1387-1405.

Kerkhoff, G., \& Zoelch, C. (1998). Disorders of visual orientation in the frontal plane in patients with visual neglect following right or left parietal lesions. Experimental Brain Research, 122, 108-120.

Lechelt, E. C., Eliuk, J., \& TANne, G. (1976). Perceptual orientational asymmetries: A comparison of visual and haptic space. Perception \& Psychophysics, 20, 463-469.

Lechelt, E. C., \& VerenKa, A. (1980). Spatial anisotropy in intramodal and cross-modal judgements of stimulus orientations: The stability of the oblique effect. Perception, 9, 581-589.

LENNIE, P. (1974). Head orientation and meridional variations in acuity. Vision Research, 14, 107-111.

Luyat, M. (1997). Verticale posturale versus verticale subjective: Une note sur l'étude de la perception de la verticale [Postural vertical and subjective vertical: A note on the study of vertical orientation perception.]. L'Année Psychologique, 97, 433-447.

Mittelstaedt, H. (1983). A new solution to the problem of the subjective vertical. Naturwissenschaften, 70, 272-281.

MittelstaedT, H. (1995). The formation of the visual and postural vertical. In T. Mergner \& F. Hlavacka (Eds.), Multisensory control of posture (pp. 147-155). New York: Plenum.

Mittelstaedt, H. (1997). Interaction of eye, head, and trunk-bound information in spatial perception and control. Journal of Vestibular Research, 7, 283-302.

Orban, G. A., Vandenbussche, E., \& Vogels, R. (1984). Human orientation discrimination tested with long stimuli. Vision Research, 24, 121-128.

PArker, D. E., \& Poston, R. L. (1984). Tilt from a head-inverted position produces displacement of visual subjective vertical in the opposite direction. Perception \& Psychophysics, 36, 461-465.

Rock, I. (1990). The frame of reference. In I. Rock (Ed.), The legacy of Solomon Asch (pp. 243-268). Hillsdale, NJ: Erlbaum.

SAARINEN, J., \& LEVI, D. (1995). Orientation anisotropy in vernier acuity. Vision Research, 35, 2449-2461.

Van Hof, M. W., \& Lagers-van Haselen, G. C. (1994). The oblique effect in the human somatic sensory system. Acta Neurobiologiae Experimentalis, 54, 259-262.

WADE, N. J. (1970). Effects of prolongated tilt on visual orientation. Quarterly Journal of Experimental Psychology, 22, 423-439.

WADE, N. J. (1992). The representation of orientation in vision. Australian Journal of Psychology, 44, 139-145.

WADE, S., \& Curthoys, I. (1997). The effect of ocular torsional position on perception of the roll-tilt of visual stimuli. Vision Research, 37, 1071-1078.

(Manuscript received April 6, 1999; revision accepted for publication July 5, 2000.) 